\title{
THE PERMANENT ANALOGUE OF THE HADAMARD DETERMINANT THEOREM
}

\author{
BY MARVIN MARCUS
}

Communicated by A. M. Gleason, April 11, 1963

1. Statement of results. In $[2 ; 3]$ it was conjectured that if $A=\left(a_{i j}\right)$ is an $n$-square positive semi-definite hermitian matrix then

$$
\operatorname{per} A \geqq \prod_{i=1}^{n} a_{i i}
$$

Here per $A$ denotes the permanent of $A: \operatorname{per} A=\sum_{\sigma} \prod_{i=1}^{n} a_{i \sigma(i)}$ where the summation is over the whole symmetric group of degree $n$. It was announced [1] and later proved [2] that $\operatorname{per}(A) \geqq \operatorname{det} A$ and the Hadamard determinant theorem suggests that the product of the main diagonal entries of $A$ in fact separates the permanent and the determinant of $A$. In this note we sketch a proof of an inequality that is substantially stronger than (1). Let $A(i)$ denote the principal submatrix of $A$ obtained by deleting row and column $i$.

Theorem. If $A$ is an $(r+1)$-square positive semi-definite hermitian matrix then

$$
(r+1) a_{11} \operatorname{per} A(1) \geqq \operatorname{per} A \geqq a_{11} \operatorname{per} A(1) .
$$

If $A$ has a zero row then (2) is equality throughout. If $A$ has no zero row then the lower equality holds if and only if $A=a_{11}+A(1)$; the upper equality holds if and only if $A$ is of rank 1.

We remark that what is true for $A(1)$ is true for any $A(i)$ because the permanent is unaltered by permutation of the rows and columns.

By an obvious induction on $r$ we have the

Corollary. If $A$ is an n-square positive semi-definite hermitian matrix then

$$
\operatorname{per} A \geqq \prod_{i=1}^{n} a_{i i}
$$

with equality if and only if $A$ has a zero row or $A$ is a diagonal matrix.

2. Proof of theorem. We outline the proof of the theorem. Let $U$ be an $n$-dimensional unitary space with inner product $(x, y)$. For $1 \leqq r \leqq n$ define $U^{(r)}$ to be the space of $r$-tensors on $U$; that is, $U^{(r)}$ is the dual space of the space of all multilinear complex valued func- 
tions of $r$-tuples of vectors from $U$. If $x_{1}, \cdots, x_{r}$ are in $U$ then $x_{1} \otimes \cdots \otimes x_{r} \in U^{(r)}$ is defined by $x_{1} \otimes \cdots \otimes x_{r}(\phi)=\phi\left(x_{1}, \cdots, x_{r}\right)$ for any multilinear functional $\phi$. Define the completely symmetric operator $S: U^{(r)} \rightarrow U^{(r)}$ by

$$
S\left(x_{1} \otimes \cdots \otimes x_{r}\right)=\frac{1}{r !} \sum_{\sigma} x_{\sigma(1)} \otimes \cdots \otimes x_{\sigma(r)}
$$

where $\sigma$ runs over the symmetric group of degree $r$. Then define the symmetric product of $x_{1}, \cdots, x_{r}$ by $x_{1} \cdots x_{r}=S\left(x_{1} \otimes \cdots \otimes x_{r}\right)$. The range of $S$ is the symmetry class of completely symmetric tensors denoted by $U_{(r)}$. It is well known that an inner product in $V_{(r)}$ is given by

$$
\left(x_{1} \cdots x_{r}, y_{1} \cdots y_{r}\right)=\frac{1}{r !} \operatorname{per}\left(\left(x_{i}, y_{j}\right)\right), \quad i, j=1, \cdots, r .
$$

Now let $G_{r, n}$ denote the totality of nondecreasing sequences of length $r$ chosen from $1, \cdots, n$ and for $\omega \in G_{r, n}$ define $\mu(\omega)$ to be the product of the factorials of the multiplicities of the distinct integers in $\omega$; e.g. $\mu(1,1,4,4,4,5)=2$ !3!. From (4) it follows that if $e_{1}, \cdots, e_{n}$ is an orthonormal (o.n.) basis in $U$ then $\sqrt{ }(r ! / \mu(\omega)) e_{\omega}, \omega \in G_{r, n}$, is an o.n. basis in $U_{(r)}$ : here $e_{\omega}=e_{\omega_{1}} \cdots e_{\omega_{r}}$.

Now let $e_{1}$ be a unit vector in $U$ and complete to an o.n. basis of $U$ with $e_{2}, \cdots, e_{n}$. Next define a linear map $T: U_{(r)} \rightarrow U_{(r+1)}$ by $T\left(x_{1} \cdots x_{r}\right)=e_{1} \cdot x_{1} \cdots x_{r}$. From (4) it follows that if no $x_{i}=0$, $i=1, \cdots, r$, then the Rayleigh quotient

$$
\frac{\left(T x_{1} \cdots x_{r}, T x_{1} \cdots x_{r}\right)}{\left(x_{1} \cdots x_{r}, x_{1} \cdots x_{r}\right)}
$$

is given by

$$
\frac{1}{r+1} \operatorname{per} A / \operatorname{per} A(1)
$$

where $A=a_{i j}$ is the Gram matrix based on the ordered set $e_{1}, x_{1}, \ldots$, $x_{r}$ : that is,

$$
\begin{array}{rlrl}
a_{i+1, j+1} & =\left(x_{i}, x_{j}\right), & 1 \leqq i, j \leqq r, \\
a_{11} & =\left(e_{1}, e_{1}\right), & & \\
a_{1 j+1} & =\bar{a}_{j+1}=\left(e_{1}, x_{j}\right), & & j=1, \cdots, r .
\end{array}
$$

Let $H=T^{*} T$, then from (5) the problem becomes equivalent to determining the maximum and minimum eigenvalues of $H$ and the cor- 
responding eigenvectors. It turns out that a matrix representation of $H$ with respect to the lexicographically ordered basis $\sqrt{ }(r ! / \mu(\omega)) e_{\omega}$, $\omega \in G_{r, n}$, is diagonal and the eigenvalues of $H$ are

$$
\frac{\mu(1, \omega)}{\mu(\omega)} \cdot \frac{1}{r+1}
$$

with corresponding eigenvectors $e_{\omega}$ : here $(1, \omega)$ designates the sequence $\left(1, \omega_{1}, \cdots, \omega_{r}\right) \in G_{r+1, n}$. The expression (6) is bounded below by $1 /(r+1)$ and takes on this value if and only if $\omega_{1}>1$. This means that the lower inequality can hold in (2) if and only if $x_{1} \cdots x_{r}$ is in the space spanned by $e_{\omega}, \omega \in G_{r, n}, \omega_{1}>1$. We then prove that this happens if and only if $\left(x_{i}, e_{1}\right)=0, i=1, \cdots, r$, i.e., that $A=a_{11}+A(1)$. Similarly, the expression (6) is bounded above by 1 and takes on this value only for the sequence $\omega_{i}=1, i=1, \cdots, r$. This means that the upper equality can hold in (2) if and only if $x_{1} \cdots x_{r}$ is a multiple of $e_{1} \cdots e_{1}$. According to [2, Theorem 3] this happens only in case $x_{i}=d_{i} e_{1}, i=1, \cdots, r$, and this leads to the condition that rank of $A$ is 1 .

\section{REFERENCES}

1. Marvin Marcus and Morris Newman, The permanent function as an inner product, Bull. Amer. Math. Soc. 67 (1961), 223-224.

2. - Inequalities for the permanent function, Ann. of Math. (2) 75 (1962), 47-62.

3. Marvin Marcus and Henryk Minc, The Pythagorean theorem in certain symmetry classes of tensors, Trans. Amer. Math. Soc. 104 (1962), 510-515.

University of California, Santa Barbara 